\title{
The structure of weakly stable minimal hypersurfaces
}

\author{
XU CHENG ${ }^{1}$, LEUNG-FU CHEUNG ${ }^{2}$ and DETANG ZHOU $^{1}$ \\ ${ }^{1}$ Instituto de Matemática, Universidade Federal Fluminense - UFF \\ Rua Mario Santos Braga s/nº, Campus do Valonguinho, 24020-140 Niterói, RJ, Brasil \\ ${ }^{2}$ Department of Mathematics, The Chinese University of Hong Kong, Shatin Hong Kong \\ Manuscript received on July 7, 2005; accepted for publication on December 26, 2005; \\ presented by MANFREDO DO CARMO
}

\begin{abstract}
In this short communication, we announce results from our research on the structure of complete noncompact oriented weakly stable minimal hypersurfaces in a manifold of nonnegative sectional curvature. In particular, a complete oriented weakly stable minimal hypersurface in $\mathbb{R}^{m}, m \geq 4$, must have only one end; any complete noncompact oriented weakly stable minimal hypersurface has only one end if the complete oriented ambient manifold $N^{m}, m \geq 7$, has nonnegative sectional curvature and Ricci curvature bounded below by a positive constant; a complete oriented weakly stable minimal hypersurface in $\mathbb{R}^{m}, m \geq 4$, with finite total scalar curvature is a hyperplane.
\end{abstract}

Key words: minimal hypersurfaces, weak stability, ends.

\section{INTRODUCTION}

Let $N^{n+1}$ be an $(n+1)$-dimensional Riemannian manifold. Let $i: M^{n} \rightarrow N^{n+1}$ be a minimal isometric immersion of a connected complete $n$-dimensional manifold $M$. $M$ is called stable if, for any $f \in C_{0}^{\infty}(M)$, it holds that

$$
\int_{M}\left\{|\nabla f|^{2}-\left(\tilde{\operatorname{Ric}}(v, v)+|A|^{2}\right) f^{2}\right\} \geq 0 .
$$

$M$ is called weakly stable if inequality (1) holds only for any $f \in C_{0}^{\infty}(M)$ satisfying the condition $\int_{M} f=0$.

The concept of (weak) stability was introduced by Barbosa, do Carmo and Eschenburg (Barbosa et al. 1988) for hypersurfaces with constant mean curvature (including the minimal case). In the current literatures, the notations of stablity on minimal and constant mean curvature hypersurfaces

Correspondence to: Xu Cheng

E-mail: xcheng@impa.br 
are different (maybe a little confusing). A hypersurface with (nonzero) constant mean curvature is called (weakly) stable if inequality (1) holds only for any $f \in C_{0}^{\infty}(M)$ with $\int_{M} f=0$ and strongly stable if inequality (1) holds for all $f \in C_{0}^{\infty}(M)$; while a minimal hypersurface is called stable if it is strongly stable in the above sense. In this paper, to avoid confusion and conform to others' notations, the term of weak stability for minimal hypersurfaces is used.

The Bernstein type problem asks whether all stable minimal hypersurfaces in $\mathbb{R}^{n+1}$ are hyperplanes when $n \leq 7$. In $n=2$, it was solved by do Carmo and Peng (do Carmo and Peng 1979); and Fischer-Colbrie and Schoen (Fischer-Colbrie and Schoen 1980). Furthermore, da Silveira (da Silveira 1987) showed that every weakly stable minimal surface in $\mathbb{R}^{3}$ is a plane. There are some results about the structure of stable minimal hypersurfaces in all $\mathbb{R}^{n+1}$. For example, $\mathrm{H}$. Cao, Y. Shen and S. Zhu (Cao et al. 1997) proved that a complete stable minimal hypersurface in $\mathbb{R}^{n+1}, n \geq 3$, must have only one end. When ambient manifolds are not necessarily Euclidean spaces, Fischer-Colbrie and Schoen (Fischer-Colbrie and Schoen 1980) obtained a classification for complete oriented stable minimal surfaces in a complete oriented 3-manifold of nonnegative scalar curvature. Recently, Li and Wang (Li and Wang 2004) gave a structure theorem on complete noncompact properly immersed stable minimal hypersurface in a complete manifold of nonnegative sectional curvature.

In this paper, we need the concepts of parabolicity and nonparabolicity. A complete manifold $\Sigma$ is called non-parabolic if it admits a positive Green function. Otherwise, $M$ is said to be parabolic. An end $E$ of $\Sigma$ is said to be non-parabolic if it admits a positive Green function with Neumann boundary condition on $\partial E$. Otherwise, it is said to be parabolic. In this paper, Ric and $\tilde{\mathrm{K}}$ denote the Ricci and sectional curvatures of the ambient manifold respectively.

\section{STATEMENT OF RESULTS}

In (X. Cheng et al., unpublished data), we discuss the global property of weakly stable minimal hypersurfaces in a Riemannian manifold. First we prove that

THEOREM 1. Let $N^{n+1}, n \geq 6$, be an ( $\left.n+1\right)$-dimensional complete oriented Riemannian manifold of nonnegative sectional curvature and Ricci curvature bounded below by a positive constant. If $M$ is a complete noncompact oriented weakly stable oriented minimal immersed hypersurface in $N$, then $M$ has only one end. This implies that a complete oriented stable immersed minimal hypersurface in such $N$ must have only one end.

The reason for the restriction on dimensions $(n \geq 6)$ is that Fischer-Colbrie and Schoen (Fischer-Colbrie and Schoen 1980) showed that there are no complete stable minimal surfaces in a 3-manifold of positive Ricci curvature; and Cheng (X. Cheng, unpublished data) proved that in the cases: $3 \leq n \leq 5$, there are no complete noncompact minimal hypersurfaces with finite index in an $(n+1)$-manifold of nonnegative sectional curvature and Ricci curvature bounded below by a positive constant. 
Next we study the structure of weakly stable minimal hypersurfaces in $\mathbb{R}^{n+1}, n \geq 3$, and obtain that

THEOREM 2. A complete oriented weakly stable minimal hypersurface in $\mathbb{R}^{n+1}, n \geq 3$, must have only one end.

COROLLARY 1. A complete oriented weakly stable immersed minimal hypersurface in $\mathbb{R}^{n+1}, n \geq 3$ with finite total scalar curvature (i.e., $\int_{M}|A|^{n}<\infty$ ) is a hyperplane.

Corollary 1 generalizes the result of Y.B. Shen and X. Zhu (Shen and Zhu 1998) that a complete stable immersed minimal hypersurface in $\mathbb{R}^{n+1}$ with finite total scalar curvature is a hyperplane. Since the catenoid type hypersurfaces in $\mathbb{R}^{n+1}(n \geq 3)$ have index 1 , the condition of Corollary 1 on index is sharp.

Finally we study the structure of weakly stable minimal hypersurfaces in a manifold of nonnegative sectional curvature and obtain

THEOREM 3. Let $N$ be a complete oriented Riemannian manifold of bounded geometry and nonnegative sectional curvature and $M$ a complete noncompact oriented weakly stable minimal hypersurface immersed in $N$ respectively. Then

(1) if $M$ is parabolic, then either it has only one end and nonnegative curvature; or it is isometric to $\mathbb{R} \times P$ with the product metric, where $P$ is a compact manifold of nonnegative curvature. In both cases, $M$ is totally geodesic;

(2) if $M$ is nonparabolic, then it has only one nonparabolic end.

We study the global behavior of minimal hypersurface with weak stability by means of harmonic function theory. A significant difference between weakly stable and stable cases lies in the choice of test functions. When we deal with weak stability, the test functions $f$ must satisfy $\int_{M} f=0$. We can successfully construct the desired test functions by using the properties of harmonic functions (Lemma 1 and Proposition 4). We also study the case of weakly stable hypersurfaces with constant mean curvature in (Cheng et al. 2005).

\section{SKETCH OF PROOFS}

The method to prove Theorems 1 and 2 is the following: Denote by $H_{D}^{\infty}(M)$ the space of bounded harmonic functions on a complete Riemannian manifold $M$ with finite Dirichlet integral. Li and Tam ( $\mathrm{Li}$ and Tam 1992, Th.2.1) showed that the number of nonparabolic ends of $M$ is bounded from above by the dimension of $H_{D}^{\infty}(M)$. Hence if we know that every end of $M$ is nonparabolic, then we obtain that the number of its ends is no more than $\operatorname{dim} H_{D}^{\infty}(M)$. Also, Li and Wang (Li and Wang 2002, Corollary 4) proved that if an end of a manifold is of infinite volume and satisfies a Sobolev type inequality, then this end must be nonparabolic. 
In order to apply the above method, we first discuss the volume growth of ends of complete noncompact submanifolds in a Riemannian manifold $N$ of bounded geometry (i.e., its sectional curvatures $\tilde{\mathrm{K}} \leq \sigma^{2}, \sigma>0$ and its injectivity radius $\left.i_{N}(p) \geq i_{0}, i_{0}>0\right)$ and obtain

PROPOSITION 1. Let $N$ be an m-dimensional manifold of bounded geometry and let $M$ be an $n$-dimensional complete noncompact manifold. Let $x: M \rightarrow N$ be an isometric immersion with mean curvature vector field bounded in norm. Then each end $E$ of $M$ has infinite volume. More precisely, the rate of volume growth of $E$ is at least linear, i.e., for any $p \in E$,

$$
\liminf _{R \rightarrow \infty} \frac{\operatorname{Vol}\left(B_{p}(R) \cap E\right)}{R}>0,
$$

where the limit is independent of the choice of $p$.

By Proposition 1, we can obtain the following Proposition 2 and Proposition 3:

PROPOSITION 2. Let $N^{m}$ be a complete simply connected manifold with nonpositive sectional curvature and let $M^{n}$ be a complete immersed minimal submanifold in $N^{m}$. If $n \geq 3$, then each end of $M$ must be nonparabolic.

PROPOSITION 3. Let $N^{n+1}$ be a complete manifold of Ricci curvature bounded below by a positive constant and $M^{n}$ be a complete noncompact immersed minimal hypersurface in $N^{n+1}$. If $M$ has finite index, then each end of $M$ must be nonparabolic.

We next consider the non-existence of nonconstant bounded harmonic functions with finite Dirichlet integral and prove the following Lemma 1.

LEMMA 1. Let $M^{n}$ be a complete noncompact weakly stable minimal hypersurface in a manifold $N^{n+1}$. If

$$
\tilde{\operatorname{Ric}}(X)+\tilde{\operatorname{Ric}}(Y)-\tilde{\mathrm{K}}(X, Y) \geq 0, X, Y \in T_{p} N, X \perp Y, \forall p \in M,
$$

then there exist no nonconstant bounded harmonic functions with finite Dirichlet integral on M.

The sketch of the proof of Lemma 1: Suppose, to the contrary, that $u$ is a nonconstant bounded harmonic function with $\int_{M}|\nabla u|^{2}<\infty$. By the properties of $u$, we can prove that $\int_{M}|\nabla u|=\infty$.

For $R>a$, take

$$
\begin{gathered}
\varphi_{1, a, R}(r)= \begin{cases}1, & \text { on } B_{p}(a), \\
\frac{a+R-r}{R}, & \text { on } B_{p}(a+R) \backslash B_{p}(a), \\
0, & \text { on } M \backslash B_{p}(a+R) ;\end{cases} \\
\varphi_{2, a, R}(r)= \begin{cases}0, & \text { on } B_{p}(a+R), \\
\frac{a+R-r}{R}, & \text { on } B_{p}(a+2 R) \backslash B_{p}(a+R), \\
-1, & \text { on } B_{p}(a+2 R+b) \backslash B_{p}(a+2 R), \\
\frac{r-(a+3 R+b)}{R}, & \text { on } B_{p}(a+3 R+b) \backslash B_{p}(a+2 R+b), \\
0, & \text { on } M \backslash B_{p}(a+3 R+b),\end{cases}
\end{gathered}
$$


where constant $b>0$ will be determined later.

For any $\epsilon>0$, by the assumption on finite Dirichlet integral, we may choose large $R$ so that $\frac{1}{R^{2}} \int_{M}|\nabla u|^{2}<\epsilon$. Define $\psi_{t, a, R}=\varphi_{1, a, R}+t \varphi_{2, a, R}, t \in[0,1]$. We have

$$
\int_{M} \psi_{0, a, R}|\nabla u| \geq \int_{B_{p}(a)}|\nabla u|>0,
$$

and

$$
\int_{M} \psi_{1, a, R}|\nabla u| \leq \int_{B_{p}(a+R)}|\nabla u|-\int_{B_{p}(a+2 R+b) \backslash B_{p}(a+2 R)}|\nabla u| .
$$

For $a$ and $R$ fixed, we may find $b$ sufficiently large, depending on $a$ and $R$ such that $\int_{M} \psi_{1, a, R}|\nabla u|<$ 0 . By the continuity of $\psi_{t, a, R}$ on $t$, there exists some $t_{0} \in(0,1)$ depending on $a$ and $R$ such that $\int_{M} \psi_{t_{0}, a, R}|\nabla u|=0$.

Since $M$ is weakly stable, $\phi=\psi_{t_{0}, a, R}|\nabla u|$ satisfies the stability inequality (1). Then, we can obtain

$$
\left.\int_{B_{p}(a)}|\nabla| \nabla u\right|^{2} \leq \frac{C}{R^{2}} \int_{M}|\nabla u|^{2}<\epsilon .
$$

By the arbitrariness of $\epsilon$ and $a,|\nabla u| \equiv$ constant. If $|\nabla u| \equiv$ constant $\neq 0$, then $u$ is a nonconstant bounded harmonic function. This says that $M$ is nonparabolic and so has infinite volume. Hence $\int_{M}|\nabla u|^{2}=\infty$, which is impossible. Therefore $|\nabla u| \equiv 0, u \equiv$ constant. This contradiction finishes the proof.

Now we can prove Theorem 2. By Lemma 1, the dimension of the space $H_{D}^{0}(M)$ is 1 . By Proposition 2, each end of $M$ must be nonparabolic. Hence $M$ must have only one end. Like the proof of Theorem 2, by Proposition 3, Lemma 1, and Theorem 0.1 in (X. Cheng, unpublished data), we obtain Theorem 1. Recall that Anderson (Anderson 1986, Th.5.2) proved that a complete minimal hypersurface in $\mathbb{R}^{n+1}(n \geq 3)$ with finite total scalar curvature and one end must be an affine-plane. Hence by the result of Anderson and Theorem 2, we obtain Corollary 1.

Finally, we study the structure of weakly stable minimal hypersurfaces in a manifold of nonnegative curvature. We have to deal with parabolic case.

\section{(I) PARABolic CASE:}

We first obtain a result on Schrödinger operator for parabolic manifolds, which may be of its independent interest.

PROPOSITION 4. Let $M$ be a complete parabolic manifold with infinity volume. Consider the operator $L=\Delta+q(x)$ on $M$ (here $q$ is a differentiable function on $M$ ). If $q(x) \geq 0$ and $q \neq \equiv$, then there exists a compactly supported piecewise smooth function $\psi$ such that $\int_{M} \psi(x)=0$ and $-\int_{M} \psi L \psi<0$.

Proposition 4 generalized the corresponding 2-dimensional result by da Silveira (da Silveira 1987). In its proof, we use the following property to construct the required test functions: Since $M$ 
is parabolic, for $p \in M$ fixed, there exist the harmonic functions $h_{r}, r>1$, on annuli $B_{p}(r) \backslash B_{p}(1)$ with the Dirichlet boundary conditions $h_{r}=1$ on $\partial B_{p}(1)$ and $h_{r}=0$ on $\partial B_{p}(r)$, satisfying $E(r)=\int_{B_{p}(r) \backslash B_{p}(1)}\left|\nabla h_{r}\right|^{2} \rightarrow 0$, as $r \rightarrow+\infty$. Proposition 4 enable us to show that

THEOREM 4. Let $N^{n+1}$ be a complete manifold of nonnegative Ricci curvature and $M$ be a complete weakly stable minimal hypersurface in $N$. If $M$ is parabolic and has infinite volume, then $M$ must be totally geodesic in N. Moreover the Ricci curvature Ric $(v, v)$ in the normal direction is identically equal to 0 along $M$ and the scalar curvature $S$ of $M$ is nonnegative.

COROLLARY 2. Let $N^{n+1}$ be a complete oriented manifold of bounded geometry and nonnegative Ricci curvature and $M$ be a complete noncompact weakly stable hypersurface minimal immersed in $N^{n+1}$. If $M$ is parabolic, then $M$ must be totally geodesic in $N$. Moreover the Ricci curvature $\operatorname{Ric}(v, v)$ in the normal direction is identically equal to 0 along $M$ and the scalar curvature $S_{M}$ is nonnegative.

THEOREM 5. Let $N$ be a complete oriented manifold of bounded geometry and nonnegative sectional curvature and $M$ be a complete noncompact weakly stable minimal hypersurface immersed in $N$. If $M$ is parabolic, then it is totally geodesic and has nonnegative sectional curvature. Further, either

(1) M has only one end; or

(2) $M=\mathbb{R} \times P$ with the product metric, where $P$ is a compact manifold of nonnegative curvature.

\section{(II) NONPARABOLIC CASE:}

In this situation, as a consequence of Lemma 1, we have

THEOREM 6. Let $N$ be a complete oriented Riemannian manifold of nonnegative bi-Ricci curvature and $M$ be a complete noncompact weakly stable minimal hypersurface immersed in N. If $M$ is nonparabolic, then it must only have one nonparabolic end.

Combining Theorem 6 and Theorem 5, we obtain Theorem 3.

\section{ACKNOWLEDGMENTS}

Xu Cheng is supported by Conselho Nacional de Desenvolvimento Científico e Tecnológio (CNPq) and Detang Zhou is partially supported by CNPq and Coordenação de Aperfeiçoamento de Pessoal de Nível Superior (CAPES).

\section{RESUMO}

Neste trabalho, anunciamos resultados de nossa investigação sobre a estrutura das hipersuperfícies mínimas completas e fracamente estáveis em um espaço ambiente de curvatura seccional não-negativa. Em particular, uma hipersuperfície mínima orientável completa e estável em $\mathbb{R}^{m}, m \geq 4$, possui apenas um fim, e uma 
superfície mínima completa orientável, fracamente estável tem apenas um fim se a variedade ambiente $N^{m}$, $m \geq 7$, tem curvatura seccional não-negativa e curatura de Ricci limitada inferiormente por uma constante positiva. Finalmente, uma hipersuperfície mínima, orientável, completa fracamente estável em $\mathbb{R}^{m}, m \geq 4$, com curvatura total infinita é um hiperplano.

Palavras-chave: hipersuperfícies mínimas, estabilidade fraca, fins.

\section{REFERENCES}

ANDERSON MT. 1986. The compactification of a minimal submanifold in Euclidean space by the Gauss map, preprint (final version in Dept. of Math. California Institute of Technology. Pasadena. CA 91125).

Barbosa JL, Do CARmo M And Eschenburg J. 1988. Stability of hypersurfaces of constant mean curvature in Riemannian manifolds. Math Z 197: 123-138.

CAO H, SHEN Y AND ZHU S. 1997. The structure of stable minimal hypersurfaces in $R^{n+1}$. Math Res Lett 1997: 637-644.

Cheng X, Cheung LF AND ZHou D. 2005. Stable constant mean curvature hypersurfaces in a Riemannian manifold (preprint).

Do CARMo M AND Peng CK. 1979. Stable complete minimal surfaces in $\mathbb{R}^{3}$ are planes. Bull Amer Math Soc 1: 903-906.

DA SILVEIRA AM. 1987. Stability of complete noncompact surfaces with constant mean curvature. Math Ann 277: 629-638.

Fischer-Colbrie D AND SCHOEn R. 1980. The structure of complete stable minimal surfaces in 3-manifolds of nonnegative scalar curvature. Comm Pure Appl Math 33: 199-211.

Li P AND TAM LF. 1992. Harmonic functions and the structure of complete manifolds. J Diff Geom 35: 359-383.

Li P AND WANG JP. 2002. Minimal hypersurfaces with finite index. Math Res Lett 9: 95-103.

Li P AND WANG JP. 2004. Stable minimal hypersurfaces in a non-negatively curved manifolds. J Reine Ang Math. (Crelles J.) 566: 215-230.

SHEN YB AND ZHU XH. 1998. On stable complete minimal hypersurfaces in $R^{n+1}$. Amer J Math 120: $103-116$. 